\title{
A perturbative approach to calculating the Casimir force in fluctuating scalar and vector fields
}

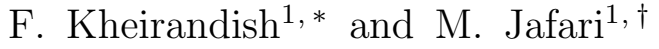 \\ ${ }^{1}$ Department of Physics, Faculty of Science, \\ University of Isfahan, Hezar-Jarib St., 81746-73441, Isfahan, Iran
}

(Dated: April 26, 2022)

\begin{abstract}
Based on a perturbative approach, a series expansion in susceptibility function of the medium is obtained for the Casimir force between arbitrary shaped objects foliated in a scalar or vector fluctuating field in arbitrary dimensions. Finite-temperature corrections are derived and the results are compared in first order with weak coupling regime in scattering method. The generalization to a massive vector field is also investigated.
\end{abstract}

PACS numbers: 12.20.Ds

Keywords: Susceptibility function; Scalar and Vector fields; Proca field; Casimir force;

*Electronic address: fardin_kh@phys.ui.ac.ir

${ }^{\dagger}$ Electronic address: jafary.marjan@gmail.com 


\section{INTRODUCTION}

The path integral is a formulation of quantum mechanics equivalent to the standard formulations, offering a new way of looking at the subject which is more intuitive than the usual approaches. Application of the path integrals are as vast as quantum mechanics itself, including the quantum mechanics of a single particle, statistical mechanics, condensed matter physics and quantum field theory [1, 2]. Quantum field theory is the quantum mechanics of continuous systems and fully developed in quantum electrodynamics which is the most successful theory in physics. The path integral in quantum field theory, both relativistic and non-relativistic, play a much more important role, for several reasons. They provide a relativity easy road to quantization and to expressions for Green's functions, which are closely related with many physical quantities. The close relation between statistical mechanics and quantum field theory is plainly visible via path integrals. Usually we are interested in a quantum field which has to be considered in the presence of a matter field described by some bosonic fields. For example, in quantum optics there are some problems where we need to quantize the electromagnetic field in the presence of some dielectrics or in calculating the effects of matter fields on Casimir forces [3 6$]$. For a consistent quantization of such fields we should include the matter fields in the process of quantization. But the main problem is that there are few situations where the interested physical quantities, like for example, the Casimir force, can be determined in a closed form and finding an effective approximation method is quite necessary [7].

The Casimir energy is the difference between the energy of the fluctuating field when then objects are present and when the objects are removed to infinity. The advent of precision experimental measurements of Casimir forces [8 12] and the possibility that they can be applied to nanoscale electromechanical devices [13, 14] has stimulated interest in developing a practical way to calculate the dependence of Casimir energies on the shapes of the objects. Many geometries have been analyzed over the years, but the case of compact objects has proved rather difficult. One way to calculate the Casimir effect is using the multiple scattering formalism, which dates back at least to the 1950. Recently Milton and et al., have noticed that the multiple scattering method can yield exact, closed form results for bodies that are weakly coupled to the quantum field [15, 16].

In the present work, we use path integrals to calculate the Casimir force between arbitrary 
shaped objects foliated in a scalar or vector fluctuating field. For this purpose, we start from a Lagrangian and develop a perturbative approach to calculating the Casimir force in the case of scalar and vector fields by taking into account the susceptibility function of the medium as the expansion parameter. The covariant formulation of the method and also its finite-temperature correction is given which may have applications in dynamical Casimir effects [17, 18]. Finally, it is shown that the results are consistent with the weak coupling limit in multiple scattering formalism as expected [15, 16, 19 24]. The present work is in fact a generalization of the previous work [27] as follows: (i) The massless scalar field in $1+1$-space time is fully investigated and an expression for Casimir force between intervals is derived. (ii) The Proca electromagnetic field is investigated and an expression for the free energy in zero and finite temperature is obtained for two arbitrarily shaped dielectrics. (iii) the approximations are improved and the results are compared with the weak coupling regime in the scattering approach.

\section{MASSLESS SCALAR FIELD}

The Lagrangian of a massless Klein-Gordon field in $N+1$-dimensional space-time $(x=$ $\left.(\mathbf{x}, t) \in \mathbb{R}^{N+1}\right)$ is the simplest field theory given by

$$
\mathcal{L}_{s}=\frac{1}{2} \partial_{\mu} \varphi(x) \partial^{\mu} \varphi(x) .
$$

As usual, let the medium be modeled by a continuum of harmonic oscillators, namely the Hopfield model [25] as follows

$$
\mathcal{L}_{r e s}=\frac{1}{2} \int_{0}^{\infty} d \omega\left(\dot{Y}_{\omega}^{2}(x)-\omega^{2} Y_{\omega}^{2}(x)\right) .
$$

This modeling can be easily generalized to a covariant form but let us first discuss the non relativistic case. The interaction between the scaler field and its medium is assumed to be linear and described by

$$
\mathcal{L}_{\text {int }}=\int_{0}^{\infty} d \omega f(\omega, \mathbf{x}) \dot{Y}_{\omega}(x) \varphi(x),
$$

where $f(\omega, \mathbf{x})$ is the coupling function between the scaler field and its medium. If the

medium has filled a finite space $\Omega$, then for $(\mathbf{x} \notin \Omega)$, there is no matter to be coupled to the field so we should set $f(\omega, \mathbf{x})=0$. Having the total Lagrangian, we can quantize the total 
system using path-integral techniques [26]. An important quantity in any field theory is the generating functional from which n-point correlation functions can be obtained by taking successive functional derivatives. Here our purpose is to find two-point correlation functions or Green's function in terms of the susceptibility of the medium. For this purpose the free generating-functional can be written as [27]

$$
\begin{aligned}
W_{0}\left[J, J_{\omega}\right] & =N e^{-\frac{1}{2 \hbar^{2}} \int d^{n} \mathbf{x} \int d t \int d^{n} \mathbf{x}^{\prime} \int d t^{\prime} J(x) G^{0}\left(x-x^{\prime}\right) J\left(x^{\prime}\right)} \\
& \times e^{-\frac{1}{2 \hbar^{2}} \int d^{n} \mathbf{x} \int d t \int d^{n} \mathbf{x}^{\prime} \int d t^{\prime} \int_{0}^{\infty} d \omega J_{\omega}(x) G_{\omega}^{0}\left(x-x^{\prime}\right) J_{\omega}\left(x^{\prime}\right)} .
\end{aligned}
$$

From the free generating-functional we can obtain the interacting generating-functional from the well known formula [26]

$$
\begin{aligned}
W\left[J, J_{\omega}\right] & =e^{\frac{i}{\hbar} \int d^{n} \mathbf{x} \int d t \int_{0}^{\infty} d \omega f(\omega, \mathbf{x})\left(\frac{\hbar}{i} \frac{\delta}{\delta J(x)}\right) \frac{\partial}{\partial t}\left(\frac{\hbar}{i} \frac{\delta}{\delta J_{\omega}(x)}\right)} W_{0}\left[J, J_{\omega}\right] \\
& =N e^{-i \hbar \int d^{n} \mathbf{x} \int d t \int_{0}^{\infty} d \omega f(\omega, \mathbf{x}) \frac{\delta}{\delta J(x)} \frac{\partial}{\partial t} \frac{\delta}{\delta J_{\omega}(x)}} \\
& \times e^{-\frac{1}{2 \hbar^{2}} \int d^{n} \mathbf{x} \int d t \int d^{n} \mathbf{x}^{\prime} \int d t^{\prime} J(x) G^{0}\left(x-x^{\prime}\right) J\left(x^{\prime}\right)} \\
& \times e^{-\frac{1}{2 \hbar^{2}} \int d^{n} \mathbf{x} \int d t \int d^{n} \mathbf{x}^{\prime} \int d t^{\prime} \int_{0}^{\infty} d \omega J_{\omega}(x) G_{\omega}^{0}\left(x-x^{\prime}\right) J_{\omega}\left(x^{\prime}\right)} .
\end{aligned}
$$

where

$$
\begin{aligned}
& G^{0}\left(x-x^{\prime}\right)=i \hbar \int \frac{d^{n} \mathbf{k} d k_{0}}{(2 \pi)^{n+1}} \frac{e^{-i k\left(\mathbf{x}-\mathbf{x}^{\prime}\right)} e^{i k_{0}\left(t-t^{\prime}\right)}}{k_{0}^{2}-\mathbf{k}^{2}+i \epsilon}, \quad(\epsilon>0), \\
& G_{\omega}^{0}\left(x-x^{\prime}\right)=i \hbar \delta^{n}\left(\mathbf{x}-\mathbf{x}^{\prime}\right) \int \frac{d k_{0}}{2 \pi} \frac{e^{i k_{0}\left(t-t^{\prime}\right)}}{\left(k_{0}\right)^{2}-\omega^{2}+i \epsilon}
\end{aligned}
$$

Now having the generating functional, the two-point function or Green's function can be obtained as

$$
G\left(x-x^{\prime}\right)=\left.\left(\frac{\hbar}{i}\right)^{2} \frac{\delta^{2}}{\delta J(x) \delta J\left(x^{\prime}\right)} W\left[J, J_{\omega}\right]\right|_{j, j_{\omega}=0} .
$$

By using Eq.(5) and after some straightforward calculations, we find the following expansion for Green's function in frequency variable

$$
\begin{gathered}
G\left(\mathbf{x}-\mathbf{x}^{\prime}, \omega\right)=G^{0}\left(\mathbf{x}-\mathbf{x}^{\prime}, \omega\right)+\int_{\Omega} d^{n} \mathbf{z}_{1} G^{0}\left(x-z_{1}, \omega\right)\left[\omega^{2} \tilde{\chi}\left(\omega, \mathbf{z}_{1}\right)\right] G^{0}\left(\mathbf{z}_{1}-\mathbf{x}^{\prime}, \omega\right)+ \\
\int_{\Omega} \int_{\Omega} d^{n} \mathbf{z}_{1} d^{n} \mathbf{z}_{2} G^{0}\left(\mathbf{x}-\mathbf{z}_{1}, \omega\right)\left[\omega^{2} \tilde{\chi}\left(\omega, \mathbf{z}_{1}\right)\right] G^{0}\left(\mathbf{z}_{1}-\mathbf{z}_{2}, \omega\right)\left[\omega^{2} \tilde{\chi}\left(\omega, \mathbf{z}_{2}\right)\right] G^{0}\left(\mathbf{z}_{2}-\mathbf{x}^{\prime}, \omega\right)+\cdots
\end{gathered}
$$

where $\tilde{\chi}(\omega, \mathbf{x})$ is the susceptibility function of the medium in frequency variable. 
By using Euler lagrange equations we find equation of motion for the fields and in particular we find a Langevin type equation for the scalar field. Specially we can show that the Green's function of the system satisfies

$$
\partial_{\mu} \partial^{\mu} G\left(\mathbf{x}-\mathbf{x}^{\prime}, t-t^{\prime}\right)+\frac{\partial}{\partial t} \int_{-\infty}^{t} d t^{\prime \prime} \chi\left(t-t^{\prime \prime}, \mathbf{x}\right) \frac{\partial}{\partial t^{\prime \prime}} G\left(\mathbf{x}-\mathbf{x}^{\prime}, t^{\prime \prime}-t^{\prime}\right)=\delta\left(\mathbf{x}-\mathbf{x}^{\prime}, t-t^{\prime}\right) .
$$

We can also show that the coupling function between the scalar field and its medium is proportional to the imaginary part of the susceptibility which is responsible for dissipation in the system

$$
f^{2}(\omega, \mathbf{x})=\frac{\omega}{\pi} \Im[\chi(\omega, \mathbf{x})]
$$

The memory function in a homogeneous medium is position independent and therefore, Eq. (9) can be solved easily in reciprocal space

$$
\tilde{G}(\mathbf{k}, \omega)=\frac{1}{\mathbf{k}^{2}-\omega^{2}-\omega^{2} \tilde{\chi}(\omega)}=\frac{1}{\mathbf{k}^{2}-\omega^{2} \frac{\epsilon(\omega)}{\epsilon_{0}}},
$$

where $\frac{\epsilon(\omega)}{\epsilon_{0}}=1+\tilde{\chi}(\omega)$ can be considered as the dielectric function of the medium. From Eq.(11) it is clear that Green's function in the presence of a homogeneous medium can be obtained from Green's function of the free space simply by substituting $\omega^{2}$ with $\omega^{2} \frac{\epsilon(\omega)}{\epsilon_{0}}$. Now Eq. (9) in frequency-space can be written as

$$
\left[\nabla^{2}-\omega^{2} \frac{\epsilon(\omega, \mathbf{x})}{\epsilon_{0}}\right] G\left(\mathbf{x}-\mathbf{x}^{\prime}, \omega\right)=\delta\left(\mathbf{x}-\mathbf{x}^{\prime}\right)
$$

In some simple geometries where dielectric function is a piecewise homogeneous function, for example for an array of parallel dielectric slabs, Eq. (9) has an exact solution, see for example [5]. The function $\epsilon(\omega, \mathbf{x})$ in a general piecewise homogeneous medium is defined by

$$
\frac{\epsilon(\omega, \mathbf{x})}{\epsilon_{0}}= \begin{cases}\epsilon(\omega), & \mathbf{x} \in \Omega \\ 1, & \mathbf{x} \notin \Omega\end{cases}
$$

where $\Omega$ is the union of regions filled with a matter with the dielectric function $\epsilon(\omega)$. In general, finding an exact solution of Eq. 12 is quite complicated or even impossible and a series solution will be useful. 


\section{COVARIANT FORMULATION}

For obtaining a covariant formulation we model the medium by a continuum of Klein-Gordon fields and write the total Lagrangian density as follows

$$
\mathcal{L}=\mathcal{L}_{s}+\mathcal{L}_{\text {res }}+\mathcal{L}_{\text {int }}
$$

where

$$
\begin{aligned}
\mathcal{L}_{m} & =\frac{1}{2} \partial_{\mu} \varphi(x) \partial^{\mu} \varphi(x), \\
\mathcal{L}_{\text {res }} & =\frac{1}{2} \int_{0}^{\infty} d \omega\left(\partial_{\mu} Y_{\omega}(x) \partial^{\mu} Y_{\omega}(x)-\omega^{2} Y_{\omega}^{2}(x)\right), \\
\mathcal{L}_{\text {int }} & =\int_{0}^{\infty} d \omega f^{\mu}(\omega, x) Y_{\omega}(x) \partial_{\mu} \varphi(x),
\end{aligned}
$$

are the Lagrangian densities of the scalar field, its medium and their interaction, respectively. Here $x=\left(x^{0}, \mathbf{x}\right)$ belongs to the Minkowski space-time $\mathbb{R}^{N+1}$. The fields $\varphi(x)$ and $Y_{\omega}(x)$ are scaler fields and the coupling function $f^{\mu}(\omega, x)$ is a vector field. From Euler-Lagrange equations we find the Langevin equation for $\varphi(x)$

$$
\partial_{\mu} \partial^{\mu} \varphi(x)+\partial_{\mu} \int d^{n+1} x^{\prime} \chi^{\mu \nu}\left(x, x^{\prime}\right) \partial_{\nu}^{\prime} \varphi\left(x^{\prime}\right)=J^{N}(x)
$$

and the Green's function of the system and the medium satisfy the following equations

$$
\begin{aligned}
\frac{i}{\hbar}\left(\partial_{\mu} \partial^{\mu}\right) G^{0}\left(x-x^{\prime}\right) & =\delta\left(x-x^{\prime}\right), \\
\frac{i}{\hbar}\left(\partial_{\mu} \partial^{\mu}+\omega^{2}\right) G_{\omega}^{0}\left(x-x^{\prime}\right) & =\delta\left(x-x^{\prime}\right) .
\end{aligned}
$$

In Eq. 16 the noise source and relativistic susceptibility are respectively defined by

$$
\begin{aligned}
J_{N}(x) & =-\partial_{\mu} \int_{0}^{\infty} d \omega f^{\mu}(\omega, x) Y_{\omega}^{N}(x), \\
\chi^{\mu \nu}\left(x-x^{\prime}\right) & =\int_{0}^{\infty} d \omega f^{\mu}(\omega, x) G_{\omega}\left(x-x^{\prime}\right) f^{\nu}\left(\omega, x^{\prime}\right) .
\end{aligned}
$$


Now using Eq. 15) we can find two-point correlation function or Green's function in terms of the susceptibility of the medium. We have

$$
\begin{aligned}
W\left[\rho, \rho_{\omega}\right] & =e^{\frac{i}{\hbar} \int d^{n+1} x \int_{0}^{\infty} d \omega f(\omega, x)\left(\frac{\delta}{\delta \rho(x)}\right) \partial_{\mu}\left(\frac{\delta}{\delta \rho \omega(x)}\right)} W_{0}\left[\rho, \rho_{\omega}\right] \\
& =N e^{-\frac{i}{\hbar} \int d^{n+1} x \int_{0}^{\infty} d \omega f(\omega, x) \frac{\delta}{\delta \rho(x)} \partial_{\mu} \frac{\delta}{\delta \rho \omega(x)}} \\
& \times e^{-\frac{1}{2 \hbar^{2}} \int d^{n+1} x \int d^{n+1} x^{\prime} \rho(x) G^{0}\left(x-x^{\prime}\right) \rho\left(x^{\prime}\right)} \\
& \times e^{-\frac{1}{2 \hbar^{2}} \int d^{n+1} x \int d^{n+1} x^{\prime}} \int_{0}^{\infty} d \omega \rho_{\omega}(x) G_{\omega}^{0}\left(x-x^{\prime}\right) \rho_{\omega}\left(x^{\prime}\right)
\end{aligned}
$$

Using this recent relation and Eq.(7) we find the following expansion for Green's function

$$
\begin{aligned}
G\left(x, x^{\prime}\right)= & G^{0}\left(x-x^{\prime}\right) \\
+ & \int_{\Omega} \int_{\Omega} d^{n+1} z_{1} d^{n+1} z_{2} G^{0}\left(x-z_{1}\right)\left[\partial_{\mu_{1}} \partial_{\mu_{2}} \chi^{\mu_{1} \mu_{2}}\left(z_{1}, z_{2}\right)\right] G^{0}\left(z_{2}-x^{\prime}\right) \\
+ & \int_{\Omega} \int_{\Omega} \int_{\Omega} \int_{\Omega} d^{n+1} z_{1} d^{n+1} z_{2} d^{n+1} z_{3} d^{n+1} z_{4} G^{0}\left(x-z_{1}\right)\left[\partial_{\mu_{1}} \partial_{\mu_{2}} \chi^{\mu_{1} \mu_{2}}\left(z_{1}, z_{2}\right)\right] G^{0}\left(z_{2}-z_{3}\right) \\
& G^{0}\left(z_{3}-z_{4}\right)\left[\partial_{\mu_{3}} \partial_{\mu_{4}} \chi^{\mu_{3} \mu_{4}}\left(z_{3}, z_{4}\right)\right] G^{0}\left(z_{4}-x^{\prime}\right)+\cdots,
\end{aligned}
$$

where susceptibility of the medium is given by Eq.(19). For a moving homogeneous material, that is a medium for which the tensor $\chi^{\mu_{1}, \mu_{2}}\left(x, x^{\prime}\right)$ is dependent only on the difference $x-x^{\prime}$, and the coupling function $f^{\mu}(\omega, x)$ is independent of $x$, the Green's function can be written in a more compact form in Fourier space as follows

$$
G(k)=G_{0}(k)\left[\mathrm{I}-G_{0}(k) k_{\mu_{1}} k_{\mu_{2}} \chi^{\mu_{1} \mu_{2}}(k)\right] .
$$

\section{PARTITION FUNCTION}

The partition function of a real scalar field in the presence of a medium is

$$
\Xi=\int D \varphi e^{\frac{i}{\hbar} S}=\int D \varphi e^{\frac{i}{\hbar} \int d^{n+1} x \mathcal{L}}
$$

where $\mathcal{L}$ is given by Eq. (1). The partition function can also be written in frequency [28]

$$
\Xi=\int D \varphi e^{-\frac{i}{2 \hbar} \int \frac{d \omega}{2 \pi} \int d^{n} \mathbf{x} \tilde{\varphi}(\mathbf{x},-\omega)\left[-\omega^{2} \frac{\epsilon(\omega, \mathbf{x})}{\epsilon_{0}}-\nabla^{2}\right] \tilde{\varphi}(\mathbf{x}, \omega)}
$$

which is much suitable for our purposes. If we make a Wick's rotation $\omega=i \nu$ in frequency

space, the action will be Euclidean and the free energy is given by $E=-\frac{\hbar}{\tau} \ln \Xi$, where $\tau$ 
is the duration of interaction and usually is taken to be quite large. Now from standard path-integral techniques we find the free energy at finite temperature $T$ as

$$
E=k_{B} T \sum_{l=0}^{\infty} \ln \operatorname{det}\left[\hat{K}\left(\nu_{l} ; \mathbf{x}, \mathbf{x}^{\prime}\right)\right]
$$

where $\nu_{l}=2 \pi l k_{B} T / \hbar$ are Matsubara frequencies and $k_{B}$ is Boltzman constant. Also $\hat{K}\left(\nu_{l} ; \mathbf{x}, \mathbf{x}^{\prime}\right)=\left[\nu_{l} \epsilon\left(i \nu_{l}, \mathbf{x}\right)-\nabla^{2}\right] \delta\left(\mathbf{x}-\mathbf{x}^{\prime}\right)$ and $\hat{K}\left(\nu_{l} ; \mathbf{x}, \mathbf{x}^{\prime}\right)=G^{-1}\left(i \nu_{l} ; \mathbf{x}, \mathbf{x}^{\prime}\right)$. Therefore

$$
E=-k_{B} T \sum_{l=0}^{\infty} \operatorname{tr} \ln \left[G\left(i \nu_{l} ; \mathbf{x}, \mathbf{x}^{\prime}\right)\right] .
$$

Now using the expansion Eq.(8), we obtain the expansion for free energy in terms of the susceptibility function as follows

$$
\begin{aligned}
E & =k_{B} T \sum_{l=0}^{\infty} \sum_{n=1}^{\infty} \frac{(-1)^{n+1}}{n} \int d^{n} \mathbf{x}_{1} \cdots d^{n} \mathbf{x}_{n} G^{0}\left(i \nu_{l} ; \mathbf{x}_{1}-\mathbf{x}_{2}\right) \ldots G^{0}\left(i \nu_{l} ; \mathbf{x}_{n}-\mathbf{x}_{1}\right) \\
& \times \chi\left(i \nu_{l}, \mathbf{x}_{1}\right) \ldots \chi\left(i \nu_{l}, \mathbf{x}_{n}\right),
\end{aligned}
$$

where $G_{0}\left(i \nu, \mathbf{x}-\mathbf{x}^{\prime}\right)$ depends on the dimension of the system.

\section{EXAMPLES}

\section{A. 1+1 Dimension}

Now let us apply the previous formalism to obtain the force induced from a fluctuating massless scalar field between two objects with susceptibilities $\chi_{1}(\omega)$ and $\chi_{2}(\omega)$ in one dimension. In this case the fluctuating field is defined over a $(1+1)$-dimensional space-time $\left(x=(\mathbf{x}, t) \in \mathbb{R}^{1+1}\right)$. In non-relativistic regime, the Green's function of the system and reservoir are respectively given by

$$
\begin{gathered}
G^{0}\left(\omega ; \mathbf{x}-\mathbf{x}^{\prime}\right)=\frac{e^{-i \omega\left|\mathbf{x}-\mathbf{x}^{\prime}\right|}}{2 \omega} \\
G_{\omega^{\prime}}^{0}\left(x-x^{\prime}\right)=\delta\left(\mathbf{x}-\mathbf{x}^{\prime}\right) \Theta\left(t-t^{\prime}\right) \frac{e^{-i \omega^{\prime}\left(t-t^{\prime}\right)}}{2 \omega^{\prime}},
\end{gathered}
$$

and in relativistic regime, these Green's functions are respectively

$$
\begin{gathered}
G^{0}\left(x, x^{\prime}\right)=-\frac{i \hbar}{2 \pi} \ln \left|x-x^{\prime}\right|, \\
G_{\omega}^{0}\left(x-x^{\prime}\right)=\frac{i \hbar}{2 \pi} K_{0}\left(\omega\left|x-x^{\prime}\right|\right),
\end{gathered}
$$


where $K_{0}(x)=\int_{0}^{\infty} d y \frac{\cos (x y)}{\sqrt{y^{2}+1}}, \quad(x>0)$, is the modified Bessel function. Here we restrict ourselves to the non-relativistic regime and assume the following form for the dielectric function

$$
\frac{\epsilon(\omega, \mathbf{x})}{\epsilon_{0}}= \begin{cases}\frac{\epsilon_{1}(\omega)}{\epsilon_{0}}, & a<\mathbf{x}<b \\ 1, & b<\mathbf{x}<c \\ \frac{\epsilon_{2}(\omega)}{\epsilon_{0}}, & c<\mathbf{x}<d .\end{cases}
$$

By inserting Eq. 28) into Eq. (27) and considering Eq. (32), up to the first relevant approximation, the free energy is obtained as

$$
E=-K_{B} T \sum_{l=1}^{\infty} \int d \mathbf{x} \int d \mathbf{x}^{\prime} \frac{e^{-2 \nu_{l}\left|\mathbf{x}-\mathbf{x}^{\prime}\right|}}{\left(2 \nu_{l}\right)^{2}} \chi\left(i \nu_{l}, \mathbf{x}\right) \chi\left(i \nu_{2}, \mathbf{x}^{\prime}\right)
$$

now for simplicity we assume that the susceptibilities are position independent, i.e., matter has been distributed in $\Omega_{1}$ and $\Omega_{2}$ intervals homogeneously, then

$$
E=-K_{B} T \sum_{l=1}^{\infty} \chi\left(i \nu_{l}\right) \chi\left(i \nu_{l}\right) \int_{\Omega_{1}} d \mathbf{x} \int_{\Omega_{2}} d \mathbf{x}^{\prime} \frac{e^{-2 \nu_{l}\left|\mathbf{x}-\mathbf{x}^{\prime}\right|}}{\left(2 \nu_{l}\right)^{2}}
$$

for further simplicity we assume that the objects have small dimensions compared to the distance between their centers of masses, therefore

$$
E=-k_{B} T \frac{(b-a)(d-c)}{4} \sum_{l=1}^{\infty}\left(\epsilon_{1}\left(i \nu_{l}\right)-1\right)\left(\epsilon_{2}\left(i \nu_{l}\right)-1\right) \frac{e^{-\nu_{l}(d+c-a-b)}}{\left(2 \nu_{l}\right)^{2}} .
$$

When the dimensions of objects are not necessarily smaller than the distance between their centers of masses we will find the following expression for the free energy

$$
\begin{aligned}
E & =-K_{B} T \sum_{l}\left[\frac{\chi_{1}^{2}\left(\nu_{l}\right)}{2 \nu_{l}}\left(-2(b-a)+\frac{1}{\nu_{l}}-\frac{e^{-2 \nu_{l}(b-a)}}{\nu_{l}}\right)\right. \\
& \left.+\frac{\chi_{2}^{2}\left(\nu_{l}\right)}{2 \nu_{l}}\left(-2(d-c)+\frac{1}{\nu_{l}}-\frac{e^{-2 \nu_{l}(d-c)}}{\nu_{l}}\right)-\frac{\chi_{1}\left(\nu_{l}\right) \chi_{2}\left(\nu_{l}\right)}{\left(\nu_{l}\right)^{2}} e^{-2 \nu_{l} r} \sinh \left(2 \nu_{l} r^{\prime}\right) \sinh \left(2 \nu_{l} r^{\prime \prime}\right)\right](36
\end{aligned}
$$

where self energies are also included. By defining

$$
\begin{aligned}
& a+b=2 r_{1}, \quad b-a=2 r^{\prime \prime} \\
& c+d=2 r_{2}, \quad d-c=2 r^{\prime}, \quad r=r_{2}-r_{1},
\end{aligned}
$$

the force induced by the fluctuating field can be obtained as

$$
F=\frac{\partial E}{\partial r}=-K_{B} T \sum_{l} \frac{2 \chi_{1}\left(\nu_{l}\right) \chi_{2}\left(\nu_{l}\right)}{\nu_{l}} e^{-2 \nu_{l} r} \sinh \left(2 \nu_{l} r^{\prime}\right) \sinh \left(2 \nu_{l} r^{\prime \prime}\right) .
$$


In zero temperature and in the case that susceptibilities are independent of frequency, the force is given by

$$
F=-\frac{\hbar \chi_{1} \chi_{2}}{2 \pi}(\Gamma[0, d-a]+\Gamma[0, c-b]-\Gamma[0, d-b]-\Gamma[0, c-a]),
$$

where $\Gamma[a, z]$ is the incomplete gamma function.

\section{B. 2+1-Dimensional space-time}

In this section we restrict ourselves to a $(2+1)$-dimensional space-time $\left(x=(\mathbf{x}, t) \in \mathbb{R}^{2+1}\right)$. In this case and in the non-relativistic regime, the Green's function of the system is given by

$$
G_{0}\left(\omega, \mathbf{x}-\mathbf{x}^{\prime}\right)=\frac{i \hbar}{2 \pi} K_{0}\left(i \omega\left|\mathbf{x}-\mathbf{x}^{\prime}\right|\right)
$$

and the form of the Green's function of the medium is the same as Eq. 229] with the difference that now $\mathbf{x}$ belongs to a two dimensional space. Now consider two objects with susceptibilities $\chi_{1}(\omega, \mathbf{x})$ and $\chi_{2}(\omega, \mathbf{x})$, the free energy in first approximation is

$$
E=-\frac{K_{B} T}{4 \pi^{2}} \sum_{l}^{\infty} \int d^{2} \mathbf{x} \int d^{2} \mathbf{x}^{\prime} K_{0}^{2}\left(\nu_{l}\left|\mathbf{x}-\mathbf{x}^{\prime}\right|\right) \chi_{1}\left(i \nu_{l}, \mathbf{x}\right) \chi_{2}\left(i \nu, \mathbf{x}^{\prime}\right)
$$

where self energies are ignored. In zero temperature the summation over the positive integer $l$ is replace by an integral according to the rule $\hbar \int_{0}^{\infty} \frac{d \nu}{2 \pi} \rightarrow K_{B} T \sum_{l=0}^{\infty}$, therefore, the free energy in zero temperature is

$$
E=-\frac{1}{32 \pi^{4}} \int d \nu \int d^{2} \mathbf{x} \int d^{2} \mathbf{x}^{\prime} K_{0}^{2}\left(\nu\left|\mathbf{x}-\mathbf{x}^{\prime}\right|\right) \chi_{1}\left(i \nu_{l}, \mathbf{x}\right) \chi_{2}\left(i \nu, \mathbf{x}^{\prime}\right) .
$$

In the case that the susceptibilities are independent of the frequency $\nu$, we obtain

$$
E=-\frac{1}{32 \pi^{3}} \int d^{2} \mathbf{x} \int d^{2} \mathbf{x}^{\prime} \frac{\chi_{1}(\mathbf{x}) \chi_{2}(\mathbf{x})}{\left|\mathbf{x}-\mathbf{x}^{\prime}\right|^{2}}
$$

Using this recent formula we can obtain for example the Casimir energy of two rings [30]. Assume that the two ring have radii $a$ and $b$, and their centers are separated by a distance $R$, Fig.(1). This situation can be shown by susceptibilities $\chi_{1}(\mathbf{x})=\chi_{1} \delta(r-a)$ and $\chi_{2}(\mathbf{x})=$ $\chi_{2} \delta\left(r^{\prime}-b\right)$ where $r$ and $r^{\prime}$ are radial coordinates in cylindrical polar coordinate system. By using Eq.43 the free energy for this geometry is given by

$$
E=\frac{-\chi_{1} \chi_{2} a b}{32 \pi^{3}} \int_{0}^{2 \pi} d \theta \int_{0}^{2 \pi} d \theta^{\prime} \frac{1}{R^{2}+a^{2}+b^{2}-2 a R \cos \theta+2 b R \cos \theta^{\prime}-2 a b \cos \left(\theta-\theta^{\prime}\right)}
$$


With a simple change in angular coordinates to $u=\theta-\theta^{\prime}$ and $v=\frac{\theta+\theta^{\prime}}{2}$ this expression can be integrated to yield the exact closed form [29]

$$
E=-\frac{\chi_{1} \chi_{2} a b}{8 \pi} \frac{1}{\sqrt{\left(R^{2}-(a-b)^{2}\right)\left(R^{2}-(a+b)^{2}\right)}},
$$

which is the same result obtained from the with weak-coupling regime in scattering formalism [30, 31]. In relativistic regime, the Green's functions of the system and reservoir respectively are

$$
\begin{aligned}
G_{0}\left(x-x^{\prime}\right) & =\frac{1}{4 \pi} \frac{1}{\left|x-x^{\prime}\right|}, \\
G_{0}^{\omega}\left(x-x^{\prime}\right) & =\frac{1}{4 \pi} \frac{e^{-i \omega\left|x-x^{\prime}\right|}}{\left|x-x^{\prime}\right|},
\end{aligned}
$$

and a similar approach can be followed for obtaining relativistic results.

\section{3+1-Dimensional space-time}

In this section we restrict ourselves to a $(3+1)$-dimensional space-time $\left(x=(\mathbf{x}, t) \in \mathbb{R}^{3+1}\right)$. In this case, in non-covariant regime, the Green's function of the system is given by

$$
G_{0}\left(\omega, \mathbf{x}-\mathbf{x}^{\prime}\right)=\frac{1}{4 \pi} \frac{e^{-i \omega\left|\mathbf{x}-\mathbf{x}^{\prime}\right|}}{\left|\mathbf{x}-\mathbf{x}^{\prime}\right|}
$$

and the free energy in zero temperature and in the first order of approximation is

$$
E=-\frac{1}{64 \pi^{3}} \int_{-\infty}^{\infty} d \nu \int d^{3} \mathbf{x} \int d^{3} \mathbf{x}^{\prime} \frac{e^{-2 \nu\left|\mathbf{x}-\mathbf{x}^{\prime}\right|} \chi_{1}(\mathbf{x}, \nu) \chi_{2}\left(\mathbf{x}^{\prime}, \nu\right)}{\left|\mathbf{x}-\mathbf{x}^{\prime}\right|^{2}}
$$

where again we have ignored the self energies which are irrelevant here. If the susceptibilities are independent of frequency the expression simplifies further to

$$
E=-\frac{1}{64 \pi^{3}} \int d^{3} \mathbf{x} \int d^{3} \mathbf{x}^{\prime} \frac{\chi_{1}(\mathbf{x}) \chi_{2}\left(\mathbf{x}^{\prime}\right)}{\left|\mathbf{x}-\mathbf{x}^{\prime}\right|^{3}}
$$

At finite temperature the integral over frequency becomes the Matsubara sum, so the energy becomes

$$
E=-\frac{K_{B} T}{32 \pi^{2}} \int d^{3} \mathbf{x} \int d^{3} \mathbf{x}^{\prime} \chi_{1}(\mathbf{x}) \chi_{2}\left(\mathbf{x}^{\prime}\right) \frac{\operatorname{coth} 2 \pi T\left|\mathbf{x}-\mathbf{x}^{\prime}\right|}{\left|\mathbf{x}-\mathbf{x}^{\prime}\right|} .
$$

As an example, we consider two spheres, of radius $a$ and $b$, respectively, with a distance between their centers $R>a+b$. The susceptibilities of the spheres are $\chi_{1}(\mathbf{x})=\chi_{1} \delta(r-a)$ and $\chi_{2}(\mathbf{x})=\chi_{2} \delta\left(r^{\prime}-b\right)$, where $r$ and $r^{\prime}$ are radial coordinates in spherical coordinate systems 
and $R$ lies along the $z$ axis of both coordinate systems, Fig.(2). Then the distance between points on the spheres is

$$
\begin{array}{r}
\left|\mathbf{x}-\mathbf{x}^{\prime}\right|=\sqrt{R^{2}+a^{2}+b^{2}-2 a b \cos \gamma-2 R\left(a \cos \theta-b \cos \theta^{\prime}\right)}, \\
\cos \gamma=\cos \theta \cos \theta^{\prime}+\sin \theta \sin \theta^{\prime} \cos \left(\phi-\phi^{\prime}\right) .
\end{array}
$$

By inserting Eq. (51) into Eq. (49) energy in zero temperature is given by

$$
E=-\frac{\chi_{1} \chi_{2} a b}{16 \pi R} \ln \frac{1-(a-b)^{2} / R^{2}}{1+(a+b)^{2} / R^{2}}
$$

which is the same result obtained from the scattering method [31].

\section{ELECTROMAGNETIC FIELD}

Now we find the Green's function of the electromagnetic field in the presence of some dielectrics from which the Casimir force in quite complicated geometries can be obtained approximately. For this purpose, the total Lagrangian density can be written in Coulomb gauge $\nabla \cdot \mathbf{A}=0, A^{0}=0$, as follows 32

$$
\mathcal{L}=\frac{1}{2}\left(\mathbf{E}^{2}-\mathbf{B}^{2}\right)+\frac{1}{2} \int_{0}^{\infty} d \omega\left(\dot{\mathbf{Y}}_{\omega}^{2}(x)-\omega^{2} \mathbf{Y}_{\omega}^{2}(x)\right)+\int d \omega f(\omega, \mathbf{x}) \mathbf{A} . \dot{\mathbf{Y}}_{\omega}
$$

Now the interacting generating functional is given by

$$
\begin{aligned}
W & =\int D[\mathbf{A}] \prod_{\omega} D\left[\mathbf{Y}_{\omega}\right] \exp \frac{i}{\hbar} \int d^{4} x\left[-\frac{1}{2} A_{\mu} \hat{K}_{i j} A_{j}-\int_{0}^{\infty} d \omega \frac{1}{2} Y_{\omega, i}\left(\partial_{t}^{2}+\omega^{2}\right) \delta_{i j} Y_{\omega, j}\right. \\
& \left.+\int_{0}^{\infty} d \omega f(\omega, \mathbf{x}) A_{i} \dot{Y}_{\omega, i}+J_{i} A_{i}+\int_{0}^{\infty} d \omega J_{\omega, i} Y_{\omega, i}\right],
\end{aligned}
$$

where the kernel $\hat{K}_{i j}$ is defined by

$$
\hat{K}_{i j}=\left(\frac{\partial_{0}^{2}}{c^{2}}-\nabla^{2}\right) \delta_{i, j}-\partial_{i} \partial_{j}
$$

Now from the well known relation

$$
G_{i j}\left(x, x^{\prime}\right)=\left.\left(\frac{\hbar}{i}\right)^{2} \frac{\delta^{2}}{\delta J_{i}(x) \delta J_{j}\left(x^{\prime}\right)} W\left[j, j_{\omega}\right]\right|_{j, j_{\omega}=0},
$$

and following the same process we did for the scaler case, we obtain the following expansion for Green's function in frequency

$$
\begin{array}{r}
G_{i j}\left(\mathbf{x}-\mathbf{x}^{\prime}, \omega\right)=G_{i j}^{0}\left(\mathbf{x}-\mathbf{x}^{\prime}, \omega\right)+\int_{\Omega} d^{3} \mathbf{z}_{1} G_{i l}^{0}\left(\mathbf{x}-\mathbf{z}_{1}, \omega\right)\left[\omega^{2} \tilde{\chi}\left(\omega, \mathbf{z}_{1}\right)\right] G_{l j}^{0}\left(\mathbf{z}_{1}-\mathbf{x}^{\prime}, \omega\right)+ \\
\int_{\Omega} \int_{\Omega} d^{3} \mathbf{z}_{1} d^{3} \mathbf{z}_{2} G_{i l}^{0}\left(\mathbf{x}-\mathbf{z}_{1}, \omega\right)\left[\omega^{2} \tilde{\chi}\left(\omega, \mathbf{z}_{1}\right)\right] G_{l m}^{0}\left(\mathbf{z}_{1}-\mathbf{z}_{2}, \omega\right)\left[\omega^{2} \tilde{\chi}\left(\omega, \mathbf{z}_{2}\right)\right] G_{m j}^{0}\left(\mathbf{z}_{2}-\mathbf{x}^{\prime}, \omega\right)+\cdots
\end{array}
$$


It can be easily shown that Green's function (57) satisfies the follows equation [27]

$$
\left[-\frac{\omega^{2}}{c^{2}} \epsilon(\omega, \mathbf{x}) \delta_{i j}-\nabla^{2} \delta_{i j}+\partial_{i} \partial_{j}\right] G_{i j}\left(\mathbf{x}, \mathbf{x}^{\prime}, \omega\right)=\delta^{3}\left(\mathbf{x}-\mathbf{x}^{\prime}\right) \delta_{i j}
$$

where $\frac{\epsilon(\omega)}{\epsilon_{0}}=\chi(\omega)+1$. A similar approach can be followed to find the partition function in terms of the susceptibility of the medium as follows

$$
\begin{aligned}
E & =k_{B} T \sum_{l=0}^{\infty} \sum_{n=1}^{\infty} \frac{(-1)^{n+1}}{n} \int d^{3} \mathbf{x}_{1} \cdots d^{3} \mathbf{x}_{n} G_{i_{1} i_{2}}^{0}\left(i \nu_{l} ; \mathbf{x}_{1}-\mathbf{x}_{2}\right) \ldots G_{i_{n} i_{1}}^{0}\left(i \nu_{l} ; \mathbf{x}_{n}-\mathbf{x}_{1}\right) \\
& \times \chi\left(i \nu_{l}, \mathbf{x}_{1}\right) \cdots \chi\left(i \nu_{l}, \mathbf{x}_{n}\right),
\end{aligned}
$$

where the free Green's function $G_{i j}^{0}\left(\mathbf{x}-\mathbf{x}^{\prime}, i \nu_{l}\right)$ satisfies Eq.58) with $\epsilon(\omega, \mathbf{x})=1$ and $\omega=i \nu_{l}$. By defining $\mathbf{r}=\mathbf{x}-\mathbf{x}^{\prime}$, we find

$$
G_{i j}^{0}\left(\mathbf{r}, i \nu_{l}\right)=\frac{\nu_{l}^{2}}{c^{2}} \frac{e^{-\frac{\nu_{l} r}{c}}}{4 \pi r}\left[\delta_{i j}\left(1+\frac{c}{\nu_{l} r}+\frac{c^{2}}{\nu_{l}^{2} r^{2}}\right)-\frac{r_{i} r_{j}}{r^{2}}\left(1+\frac{3 c}{\nu_{l} r}+\frac{3 c^{2}}{\nu_{l}^{2} r^{2}}\right)\right]+\frac{1}{3} \delta_{i j} \delta^{3}(\mathbf{r}) .
$$

As an example of the application of Eq.(59), let us find the interaction energy of a system composed of two dielectrics with volumes $V_{1}$ and $V_{2}$ and the susceptibilities $\chi_{1}$ and $\chi_{2}$, respectively. The first relevant nonzero term corresponds to $n=2$, therefore

$$
E=-\frac{1}{2} k_{B} T \sum_{l=0}^{\infty} \int_{V_{1}} \int_{V_{2}} d^{3} \mathbf{x} d^{3} \mathbf{x}^{\prime} G_{i j}^{0}\left(\mathbf{x}-\mathbf{x}^{\prime}, i \nu_{l}\right) G_{j i}^{0}\left(\mathbf{x}^{\prime}-\mathbf{x}, i \nu_{l}\right) \chi_{1}\left(i \nu_{l}, \mathbf{x}\right) \chi_{2}\left(i \nu_{l}, \mathbf{x}^{\prime}\right) .
$$

Inserting the Green's function (60) into 61 , we find

$$
E=-k_{B} T \sum_{l=0}^{\infty} \int_{V_{1}} \int_{V_{2}} d^{3} \mathbf{x} d^{3} \mathbf{x}^{\prime} \chi_{1}\left(i \nu_{l}, \mathbf{x}\right) \chi_{2}\left(i \nu_{l}, \mathbf{x}^{\prime}\right) h\left(\nu_{l},\left|\mathbf{x}-\mathbf{x}^{\prime}\right|\right)
$$

where we have defined

$$
h\left(\nu_{l},\left|\mathbf{x}-\mathbf{x}^{\prime}\right|\right)=\frac{e^{-\frac{2 \nu_{l}}{c}\left|\mathbf{x}-\mathbf{x}^{\prime}\right|}}{8 \pi^{2}}\left\{\frac{\left(\frac{\nu_{l}}{c}\right)^{4}}{\left|\mathbf{x}-\mathbf{x}^{\prime}\right|^{2}}+\frac{2\left(\frac{\nu_{l}}{c}\right)^{3}}{\left|\mathbf{x}-\mathbf{x}^{\prime}\right|^{3}}+\frac{5\left(\frac{\nu_{l}}{c}\right)^{2}}{\left|\mathbf{x}-\mathbf{x}^{\prime}\right|^{4}}+\frac{6 \frac{\nu_{l}}{c}}{\left|\mathbf{x}-\mathbf{x}^{\prime}\right|^{5}}+\frac{3}{\left|\mathbf{x}-\mathbf{x}^{\prime}\right|^{6}}\right\} .
$$

In zero temperature, the summation over the positive integer $l$ is replaced by an integral, therefore for the case that susceptibilities are independent of frequency, the free energy is given by

$$
E=-\frac{23}{64 \pi^{3}} \int d^{3} \mathbf{x} \int d^{3} \mathbf{x}^{\prime} \frac{\chi_{1}(\mathbf{x}) \chi_{2}\left(\mathbf{x}^{\prime}\right)}{\left|\mathbf{x}-\mathbf{x}^{\prime}\right|^{7}}
$$

For example for two spheres of radii $a$ and $b$, the distance between their centers $R>a+b$, with susceptibilities $\chi_{1}(x)=\chi_{1} \delta(r-a)$ and $\chi_{2}(\mathbf{x})=\chi_{2} \delta\left(r^{\prime}-b\right)$, where $r$ and $r^{\prime}$ are radial coordinates in spherical coordinate systems, the free energy from Eq. 64 is as follows

$$
E=-\frac{23 \chi_{1} \chi_{2} a^{2} b^{2}}{64 \pi^{3}} \int d \Omega \int d \Omega^{\prime} \frac{1}{\left|\mathbf{x}-\mathbf{x}^{\prime}\right|^{7}}
$$


Now we use the following formula [?]

$$
\int d \Omega d \Omega^{\prime}\left|\mathbf{x}-\mathbf{x}^{\prime}\right|^{p}=(4 \pi)^{2} R^{p} P_{p}(\hat{a}, \hat{b})
$$

where $R$ is the distance between the centers of the two non-overlapping spheres of radii $a$ and $b$, respectively. Here $\hat{a}=a / R$ and $\hat{b}=b / R$, and $P_{p}(\hat{a}, \hat{b})$ can in general be represented by the infinite series

$$
\begin{array}{r}
P_{p}(\hat{a}, \hat{b})=\sum_{n=0}^{\infty} \frac{2}{(2 n+2) !} \frac{\Gamma(2 n-p-1)}{\Gamma(-p-1)} Q_{n}(\hat{a}, \hat{b}), \\
Q_{n}=\frac{1}{2} \sum_{m=0}^{n}\left(\begin{array}{c}
2 n+2 \\
2 m+1
\end{array}\right) \hat{a}^{2(n-m)} \hat{b}^{2 m} .
\end{array}
$$

We can easily see the following recursion relation holds:

$$
P_{p-1}(\hat{a}, \hat{b})=\frac{R^{-p}}{1+p} \frac{\partial}{\partial R} R^{p+1} P_{p}(\hat{a}, \hat{b}), \quad P_{-1}=1 .
$$

Using this recent relation, the free energy in zero temperature can be written as

$$
E_{0}=-\frac{23 \chi_{1} \chi_{2} a^{2} b^{2}}{4 \pi R^{7}} P_{-7}
$$

where

$$
\begin{aligned}
P_{-7} & =\frac{-2\left(\hat{a}^{2}-\hat{b}^{2}\right)^{4}\left(\hat{a}^{2}+\hat{b}^{2}-5\right)+\left(\hat{a}^{2}-\hat{b}^{2}\right)^{2}\left(52\left(\hat{a}^{2}+\hat{b}^{2}-44\right)-24\left(\hat{a}^{2}+\hat{b}^{2}\right)^{2}\right)}{10\left(\hat{a}^{4}+\left(\hat{b}^{2}-1\right)^{2}-2 \hat{a}^{2}\left(\hat{b}^{2}+1\right)\right)^{4}} \\
& +\frac{2\left(5-5\left(\hat{a}^{2}+\hat{b}^{2}\right)+8\left(\hat{a}^{2}+\hat{b}^{2}\right)^{2}-4\left(\hat{a}^{2}+\hat{b}^{2}\right)^{3}\right)}{10\left(\hat{a}^{4}+\left(\hat{b}^{2}-1\right)^{2}-2 \hat{a}^{2}\left(\hat{b}^{2}+1\right)\right)^{4}} .
\end{aligned}
$$

In non zero temperature and first order of correction, the free energy is

$$
E=-\frac{23 \chi_{1} \chi_{2} a^{2} b^{2}}{4 \pi R^{7}} P_{-7}(\hat{a}, \hat{b})-\frac{6 k_{B} T \chi_{1} \chi_{2} a^{2} b^{2}}{R^{6}} P_{-6}(\hat{a}, \hat{b}),
$$

where

$$
P_{-6}(\hat{a}, \hat{b})=\frac{1}{4 \hat{a} \hat{b}}\left[\frac{1}{(\hat{a}-\hat{b}-1)^{3}}-\frac{1}{(\hat{a}+\hat{b}-1)^{3}}-\frac{1}{(\hat{a}-\hat{b}+1)^{3}}+\frac{1}{(\hat{a}+\hat{b}+1)^{3}}\right] .
$$

It can be shown that this results are equivalent with the finite temperature weak coupling regime in scattering formalism [29, 31]. 


\section{A. Covariant formulation}

For a covariant formulation we consider the following Lorentz invariant Lagrangian density in Lorentz gauge

$$
L(x)=-\frac{1}{4} F_{\mu, \nu} F^{\mu, \nu}+\frac{1}{2} \int_{0}^{\infty} d \omega\left[\partial_{\mu} Y_{\omega} \partial^{\mu} Y_{\omega}-\omega^{2} Y_{\omega}^{2}\right]+\int_{0}^{\infty} d \omega f^{\mu \nu}(\omega, x) Y_{\omega} \partial_{\mu} A_{\nu},
$$

where $F^{\mu, \nu}=\partial_{\mu} A_{\nu}-\partial^{\nu} A^{\mu}$ and $f^{\mu, \nu}(\omega, x)$ is an antisymmetric coupling tensor which couples electromagnetic field to the medium. From Euler-Lagrange equations we can show that Green's function satisfies the following equation [27]

$$
\square G_{\mu \nu}\left(x-x^{\prime}\right)-\int d^{4} x^{\prime \prime} g_{\mu \delta} \partial_{\gamma} \partial_{\alpha}^{\prime \prime} \chi^{\gamma \delta \alpha \beta}\left(x, x^{\prime \prime}\right) G_{\beta \nu}\left(x^{\prime \prime}-x\right)=g_{\mu \nu} \delta^{4}\left(x-x^{\prime}\right),
$$

where the susceptibility tensor $\chi^{\nu \mu \alpha \beta}\left(x, x^{\prime}\right)$ is defined by

$$
\chi_{\nu \mu \alpha \beta}\left(x, x^{\prime}\right)=\int_{0}^{\infty} d \omega f^{\mu \nu}(\omega, x) G_{\omega}^{0}\left(x-x^{\prime}\right) f^{\alpha \beta}\left(\omega, x^{\prime}\right) .
$$

For Green's function we find the following expansion in term of the susceptibility tensor

$$
\begin{array}{r}
G_{\mu \nu}\left(x, x^{\prime}\right)=G_{\mu, \nu}^{0}\left(x-x^{\prime}\right)+\int d^{4} z_{1} d^{4} z_{2} G_{\mu \nu_{1}}^{0}\left(x-z_{1}\right) \Gamma^{\nu_{1} \nu_{2}}\left(z_{1}, z_{2}\right) G_{\nu_{2} \nu}^{0}\left(z_{2}-x^{\prime}\right) \\
\int d^{4} z_{1} \ldots d^{4} z_{4} G_{\mu \nu_{1}}^{0}\left(x-z_{1}\right) \Gamma^{\nu_{1} \nu_{2}}\left(z_{1}, z_{2}\right) G_{\nu_{2} \nu_{3}}^{0}\left(z_{2}-z_{3}\right) \Gamma^{\nu_{3} \nu_{4}}\left(z_{3}, z_{4}\right) G_{\nu_{4} \nu}^{0}\left(z_{4}-x^{\prime}\right)+\cdots
\end{array}
$$

where we have defined $\Gamma^{\nu_{1} \nu_{2}}\left(z_{1}, z_{2}\right)=\partial_{\mu_{1}} \partial_{\mu_{2}} \chi^{\mu_{1} \nu_{1} \mu_{2} \nu_{2}}\left(z_{1}, z_{2}\right)$. If we are given the susceptibility tensor of the medium, we can find the Green's function perturbatively in terms of the susceptibility. Having Green's function, we can investigate for example the dynamical energy configurations, which is closely related to the dynamical Casimir effects.

\section{PROCA ELECTROMAGNETIC FIELD}

The Proca equation is a relativistic wave equation for a massive spin-1 particle. The weak interaction is transmitted by such kind of vector bosons [26, 33]. The transition from the massless electromagnetic field to a field of massive vector bosons is easily done. In contrast to the electromagnetic field, the massive spin-1 field will automatically satisfy the Lorentz condition $\partial_{\nu} A^{\nu}=0$ and the Lorentz condition has become a condition of consistency for the Proca field. Therefore the Lagrangian density of the system is given by

$$
\mathcal{L}=-\frac{1}{2} F_{\mu \nu} F^{\mu \nu}+\frac{m^{2} c^{2}}{2 \hbar} A_{\mu} A^{\mu}+\frac{1}{2} \int_{0}^{\infty} d \omega\left(\dot{\mathbf{Y}}_{\omega}^{2}(x)-\omega^{2} \mathbf{Y}_{\omega}^{2}(x)\right)+\int d \omega f(\omega, \mathbf{x}) \mathbf{A} . \dot{\mathbf{Y}}_{\omega}
$$


The interacting generating functional can be written in terms of the vector potential and the medium fields as

$$
\begin{aligned}
W & =\int D[\mathbf{A}] \prod_{\omega} D\left[\mathbf{Y}_{\omega}\right] \exp \frac{i}{\hbar} \int d^{4} x\left[-\frac{1}{2} A_{\mu} \hat{K}_{\mu \nu} A_{\nu}-\int_{0}^{\infty} d \omega \frac{1}{2} Y_{\omega, \mu}\left(\partial_{t}^{2}+\omega^{2}\right) \delta_{\mu \nu} Y_{\omega, \nu}\right. \\
& \left.+\int_{0}^{\infty} d \omega f(\omega, \mathbf{x}) A_{\mu} \dot{Y}_{\omega, \mu}+J_{\mu} A_{\mu}+\int_{0}^{\infty} d \omega J_{\omega, \mu} Y_{\omega, \mu}\right]
\end{aligned}
$$

where the kernel $\hat{K}_{\mu \nu}$ is defined by

$$
\hat{K}_{\nu \mu}=\left[\frac{\partial_{0}^{2}}{c^{2}}-\nabla^{2}\right] \delta_{\mu \nu}-\partial_{\mu} \partial_{\nu}-\frac{m^{2} c^{2}}{\hbar^{2}} \delta_{\mu \nu}
$$

Now from the well known relation

$$
G_{\nu \mu}\left(x, x^{\prime}\right)=\left.\left(\frac{\hbar}{i}\right)^{2} \frac{\delta^{2}}{\delta J_{\nu}(x) \delta J_{\mu}\left(x^{\prime}\right)} W\left[j, j_{\omega}\right]\right|_{j, j_{\omega}=0},
$$

and following the same process we did for the scaler field, we obtain the following expansion for Green's function in frequency variable

$$
\begin{gathered}
G_{\mu \nu}\left(\mathbf{x}-\mathbf{x}^{\prime}, \omega\right)=G_{\mu \nu}^{0}\left(\mathbf{x}-\mathbf{x}^{\prime}, \omega\right)+\int_{\Omega} d^{3} \mathbf{z}_{1} G_{\mu \alpha}^{0}\left(x-z_{1}, \omega\right)\left[\omega^{2} \tilde{\chi}\left(\omega, \mathbf{z}_{1}\right)\right] G_{\alpha \nu}^{0}\left(\mathbf{z}_{1}-\mathbf{x}^{\prime}, \omega\right)+ \\
\int_{\Omega} \int_{\Omega} d^{3} \mathbf{z}_{1} d^{3} \mathbf{z}_{2} G_{\mu \alpha}^{0}\left(\mathbf{x}-\mathbf{z}_{1}, \omega\right)\left[\omega^{2} \tilde{\chi}\left(\omega, \mathbf{z}_{1}\right)\right] G_{\alpha \beta}^{0}\left(\mathbf{z}_{1}-\mathbf{z}_{2}, \omega\right)\left[\omega^{2} \tilde{\chi}\left(\omega, \mathbf{z}_{2}\right)\right] G_{\beta \nu}^{0}\left(\mathbf{z}_{2}-\mathbf{x}^{\prime}, \omega\right)+\cdots
\end{gathered}
$$

Now using Euler-lagrange Equations, we find the equation of motion for the vector potential as

$$
\hat{K}_{\mu \nu} A_{\nu}+\frac{\partial}{\partial t} \int_{0}^{\infty} \frac{d \omega^{\prime}}{2 \pi} \tilde{\chi}\left(\omega^{\prime}, \mathbf{x}\right) \int d t^{\prime} e^{i \omega^{\prime}\left(t-t^{\prime}\right)} \frac{\partial}{\partial t^{\prime}} A_{\nu}\left(t^{\prime}\right)=\int_{0}^{\infty} d \omega f(\omega, \mathbf{x}) \dot{Y}_{\omega, \nu}^{N}(\omega, \mathbf{x}),
$$

where $Y_{\omega, i}^{N}$ is as a noise or fluctuating field which does not affect the Green's function. The Green's function of the above equation satisfies

$$
\left[-\left(\frac{\omega^{2}}{c^{2}} \epsilon(\omega, x)+\frac{m^{2} c^{2}}{\hbar^{2}}\right) \delta_{\mu \nu}-\nabla^{2} \delta_{\mu \nu}-\partial_{\mu} \partial_{\nu}\right] G_{\mu \nu}\left(x, x^{\prime}, \omega\right)=\delta^{3}\left(x-x^{\prime}\right) \delta_{\mu \nu}
$$

where $\epsilon(\omega, \mathbf{x})=\epsilon_{0}[1+\tilde{\chi}(\omega, \mathbf{x})]$. It can be easily shown that Green's function 81) satisfies in Eq.83). A similar approach can be followed to find the free energy in terms of the susceptibility of the medium as

$$
\begin{aligned}
E & =k_{B} T \sum_{l=0}^{\infty} \sum_{n=1}^{\infty} \frac{(-1)^{n+1}}{n} \int d^{3} \mathbf{x}_{1} \cdots d^{3} \mathbf{x}_{n} G_{\nu_{1} \nu_{2}}^{0}\left(i \nu_{l} ; \mathbf{x}_{1}-\mathbf{x}_{2}\right) \cdots G_{\nu_{n} \nu_{1}}^{0}\left(i \nu_{l} ; \mathbf{x}_{n}-\mathbf{x}_{1}\right) \\
& \times \chi\left(i \nu_{l}, \mathbf{x}_{1}\right) \cdots \chi\left(i \nu_{l}, \mathbf{x}_{n}\right),
\end{aligned}
$$


where the free Green's function $G_{\mu \nu}^{0}\left(\mathbf{x}-\mathbf{x}^{\prime}, i \nu_{l}\right)$ satisfies in Eq. 83 with $\epsilon(\omega, \mathbf{x})=1$ and $\omega=i \nu_{l}$. By defining $\mathbf{r}=\mathbf{x}-\mathbf{x}^{\prime}$, we find

$$
G_{\mu \nu}^{0}\left(\mathbf{r}, i \nu_{l}\right)=\zeta^{2} \frac{e^{-\zeta r}}{4 \pi r}\left[\delta_{\mu \nu}\left(1+\frac{1}{\zeta r}+\frac{1}{\zeta^{2} r^{2}}\right)-\frac{r_{\mu} r_{\nu}}{r^{2}}\left(1+\frac{3}{\zeta r}+\frac{3}{\zeta^{2} r^{2}}\right)\right]+\frac{1}{3} \delta_{\mu \nu} \delta^{3}(\mathbf{r}),
$$

where $\zeta=\sqrt{\left(\frac{\nu_{l}}{c}\right)^{2}-\left(\frac{m c}{\hbar}\right)^{2}}$. As an example of applications of Eq. 84), let us find the interaction energy of a system composed of two dielectrics with the volumes $V_{1}$ and $V_{2}$ and susceptibilities $\chi_{1}$ and $\chi_{2}$, respectively. The first relevant nonzero term corresponds to $n=2$, therefore

$$
E=-\frac{1}{2} k_{B} T \sum_{l=0}^{\infty} \int_{V_{1}} \int_{V_{2}} d^{3} \mathbf{x} d^{3} \mathbf{x}^{\prime} G_{\mu \nu}^{0}\left(\mathbf{x}-\mathbf{x}^{\prime}, i \nu_{l}\right) G_{\nu \mu}^{0}\left(\mathbf{x}^{\prime}-\mathbf{x}, i \nu_{l}\right) \chi_{1}\left(i \nu_{l}, \mathbf{x}\right) \chi_{2}\left(i \nu_{l}, \mathbf{x}^{\prime}\right) .
$$

Inserting the Green's function (85) into (86), we find

$$
E=-k_{B} T \sum_{l=0}^{\infty} \int_{V_{1}} \int_{V_{2}} d^{3} \mathbf{x} d^{3} \mathbf{x}^{\prime} \chi_{1}\left(i \nu_{l}, \mathbf{x}\right) \chi_{2}\left(i \nu_{l}, \mathbf{x}^{\prime}\right) h\left(\nu_{l},\left|\mathbf{x}-\mathbf{x}^{\prime}\right|\right),
$$

where we have defined

$$
h\left(\nu_{l},\left|\mathbf{x}-\mathbf{x}^{\prime}\right|\right)=\frac{e^{-2 \zeta\left|\mathbf{x}-\mathbf{x}^{\prime}\right|}}{8 \pi^{2}}\left\{\frac{\zeta^{4}}{\left|\mathbf{x}-\mathbf{x}^{\prime}\right|^{2}}+\frac{2 \zeta^{3}}{\left|\mathbf{x}-\mathbf{x}^{\prime}\right|^{3}}+\frac{5 \zeta^{2}}{\left|\mathbf{x}-\mathbf{x}^{\prime}\right|^{4}}+\frac{6 \zeta}{\left|\mathbf{x}-\mathbf{x}^{\prime}\right|^{5}}+\frac{3}{\left|\mathbf{x}-\mathbf{x}^{\prime}\right|^{6}}\right\} .
$$

In zero temperature we have

$$
\begin{aligned}
E=-\frac{c}{32 \pi^{4}} \int_{V_{1}} \int_{V_{2}} d^{3} \mathbf{x} d^{3} \mathbf{x}^{\prime} \chi_{1}(\mathbf{x}) \chi_{2}( & \mathbf{x})\left[\left(\frac{m c}{\hbar}\right)^{5} \frac{1}{\left|\mathbf{x}-\mathbf{x}^{\prime}\right|^{2}} G_{13}^{31}\left(\left.\left(\frac{m c}{\hbar}\right)^{2}\left|\mathbf{x}-\mathbf{x}^{\prime}\right|^{2}\right|_{\frac{-5}{2}, 0, \frac{1}{2}} ^{-2}\right)\right. \\
& +2\left(\frac{m c}{\hbar}\right)^{4} \frac{1}{\left|\mathbf{x}-\mathbf{x}^{\prime}\right|^{3}} G_{13}^{31}\left(\left.\left(\frac{m c}{\hbar}\right)^{2}\left|\mathbf{x}-\mathbf{x}^{\prime}\right|^{2}\right|_{-2,0, \frac{1}{2}} ^{-\frac{3}{2}}\right) \\
& +5\left(\frac{m c}{\hbar}\right)^{3} \frac{1}{\left|\mathbf{x}-\mathbf{x}^{\prime}\right|^{4}} G_{13}^{31}\left(\left.\left(\frac{m c}{\hbar}\right)^{2}\left|\mathbf{x}-\mathbf{x}^{\prime}\right|^{2}\right|_{-\frac{3}{2}, 0, \frac{1}{2}} ^{-1}\right) \\
& +6\left(\frac{m c}{\hbar}\right)^{2} \frac{1}{\left|\mathbf{x}-\mathbf{x}^{\prime}\right|^{5}} G_{13}^{31}\left(\left.\left(\frac{m c}{\hbar}\right)^{2}\left|\mathbf{x}-\mathbf{x}^{\prime}\right|^{2}\right|_{-1,0, \frac{1}{2}} ^{-\frac{1}{2}}\right) \\
& \left.+3\left(\frac{m c}{\hbar}\right) \frac{1}{\left|\mathbf{x}-\mathbf{x}^{\prime}\right|^{6}} G_{13}^{31}\left(\left.\left(\frac{m c}{\hbar}\right)^{2}\left|\mathbf{x}-\mathbf{x}^{\prime}\right|^{2}\right|_{-\frac{1}{2}, 0, \frac{1}{2}} ^{0}\right)\right]
\end{aligned}
$$

where $G_{p, q}^{m, n}\left(\left.x\right|_{b_{1}, \ldots, b_{q}} ^{a_{1}, \ldots, a_{n}}\right)$ is the Meijer function [34]. Now for simplicity let us assume that the volumes have small dimensions compared to the distance $R$ between their centers of masses, such that $\left|r_{1}-r_{2}\right| \approx R$. Therefore

$$
\begin{aligned}
E=-\frac{c \chi_{1} \chi_{2} V_{1} V_{2}}{\pi^{3}} & {\left[\frac{23}{64 R^{7}}-\frac{m c}{\hbar} \frac{3}{16 R^{6}}+\left(\frac{m c}{\hbar}\right)^{2} \frac{3}{64 R^{5}}(-3+2 \gamma+2 \log R)\right.} \\
& \left.+\left(\frac{m c}{\hbar}\right)^{3} \frac{1}{48 R^{4}}+\left(\frac{m c}{\hbar}\right)^{4} \frac{6}{R^{3}}(-1+4 \gamma+4 \log R)+\cdots\right],
\end{aligned}
$$

where $\gamma=0.577$. The first term gives the normal Casimir attractive force and the lowest order finite-mass correction tends to reduce the effect. 


\section{CONCLUSION}

Quantum field theory in the presence of a medium is investigated for a scalar field and massless and massive vector fields in different dimensions. Finite temperature corrections are considered and series expansions for corresponding Greens functions and free energies are obtained in terms of the susceptibility function of the medium. Some illustrative examples are given showing the applicability and the efficiency of the method. It is shown that the Casimir energy in first order approximation in the present method is equivalent with the weak coupling regime in the scattering method. The covariant formulation of the problem is presented. Finally, the Casimir energy in the fluctuating Proca field is investigated.

[1] W. Greiner and J. Reinhardt, Quantum Electrodynamics, 4th ed. (Springer-Verlag, Berlin, Heidelberg)(2009).

[2] H. Kleinert, Path Integrals in Quantum Mechanics, Statistics, Polymer Physics, and Financial Markets, 5th ed. (World Scientific, Singapore)(2009).

[3] W. Vogel and D.-G. Welsch, Quantum Optics, 3rd ed. (Wiley- VCH Verlag GmbH and Co., Weinheim)(2006).

[4] F. Kheirandish and M. Amooshahi, Phys. Rev. A 74, 042102(2006); M. Amooshahi and F. Kheirandish, ibid. 76, 062103 (2007); F. Kheirandish and M. Soltani, ibid. 78, 012102 (2008).

[5] R. Matloob and H. Falinejad, Phys. Rev. A 64, 042102 (2001); R. Matloob, A. Keshavarz, and D. Sedighi, ibid. 60, 3410 (1999).

[6] F. Kheirandish, M. Soltani, and J. Sarabadani, Phys. Rev. A 81, 052110 (2010).

[7] W. Greiner and J. Reinhardt, Field Quantization (Springer-Verlag, Berlin, Heidelberg)(1996).

[8] F. Chen, G. L. Klimchitskaya, V. M. Mostepanenko, and U. Mohideen, Phys. Rev. Lett. 97, $170402(2006)$.

[9] R. S. Decca, D. Lopez, E. Fischbach, G. L. Klimchitskaya, D. E. Krause, and V. M. Mostepanenko, Phys. Rev. D 75, 077101 (2007).

[10] F. Chen, G. L. Klimchitskaya, V. M. Mostepanenko, and U. Mohideen, Phys. Rev. B 76, 035338 (2007).

[11] J. N. Munday and F. Capasso, Phys. Rev. A 75, 060102(R)(2007). 
[12] D. E. Krause, R. S. Decca, D. Lopez, and E. Fischbach, Phys. Rev. Lett. 98, 050403 (2007).

[13] H. B. Chan, V. A. Aksyuk, R. N. Kleiman, D. J. Bishop, and F. Capasso, Phys. Rev. Lett. 87, 211801 (2001).

[14] F. Capasso, J. N. Munday, D. Iannuzzi, and H. B. Chan, IEEE J. Quantum Electron. 13, 400 (2007).

[15] K. A. Milton,j. Wagner, J. Phys. A 41, 155402 (2008).

[16] K. A. Milton, j. Wagner, Phys. Rev. D 77, 045005 (2008).

[17] M. Amooshahi, Eur. Phys. J. D 54, 115 (2009).

[18] R. Golestanian and M. Kardar, Phys. Rev. A 58, 1713 (1998).

[19] M. T. Homer Reid, A. W. Rodriguaz, J. White, and S. G. Johnson, Phys. Rev. Lett. 103, 040401 (2009).

[20] J. Wagner, K. A. Milton, P. Parashar, Phys. Rev. Lett. 101, 160402 (2008).

[21] T. Emig and R. L. Jaffe, J. Phys. A: Math. Theor. 41, 164001 (2008).

[22] T. Emig,N. Graham, R. L. Jaffe and M. Kardar, Phys. Rev. D 77, 025005 (2008).

[23] K. A. Milton, J. Wagner, P. Parashar, I. C. Pelaez, I. Brevik and S. A. Ellingsen, Springer Proceedings in Physics.137, 99 (2011).

[24] K. A. Milton1, P. Parashar, J. Wagner1, and I. C. Pelaez,J. Vac. Sci. Technol. B 28, C4A8 (2010);

[25] J. J. Hopfield, Phys. Rev.112, 1555(1958).

[26] W. Greiner J. Richardt,Field quantization (Springer-Verlag, Berlin, Heidelberg)(1996).

[27] F. Kheirandish, S. Salimi, Phys. Rev. A 84, 062122 (2011).

[28] J. I. Kapusta, Finite-Temperature Field Theory (Cambridge University Press, Cambridge)(1989).

[29] K. A. Milton and J. Wagner, J. Phys. A: Math. Theor. 41, 155402 (2008).

[30] J. Wagner, K. A. Milton, P. Parashar, J. Phys: Conf. Ser. 161012022 (2008).

[31] K. A. Milton, J. Wagner, P. Parashar, arXive:0909.0977v3 (2010).

[32] F. Kheirandish and M. Soltani, Phys. Rev. A 78, 012102 (2008).

[33] C. Itzykson and J. B. Zuber, Quantum field theory (McGraw-Hill, N.Y)(1980).

[34] I. S. Gradshteyn and I. M. Ryzhik, Table of Integrals, Series, and Products (Academic Press, Elsevier)(2007). 

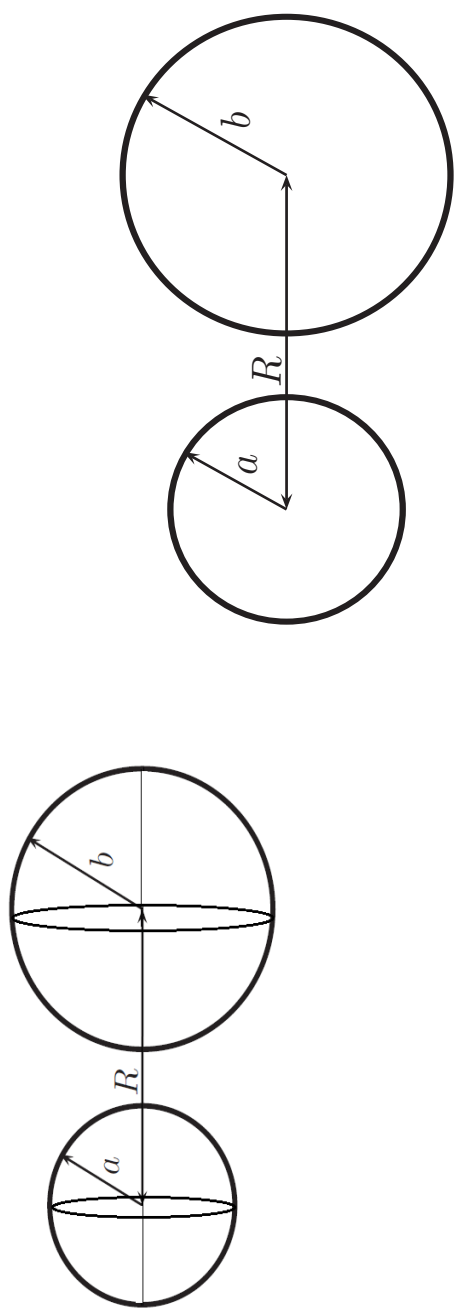

Fig.1: Two cylinders of radii $a$ and $b$, their centers are separated by a distance $R>a+b$. Fig.2: Two spheres of radii $a$ and $b$, their centers are separated by a distance $R>a+b$. 